\title{
Safety of pregnancy in uterine fibroids
}

\author{
Maha Alhainiah ${ }^{1 *}$, Elaf Aljifry ${ }^{1}$, Ayman Alghamdi $^{1}$, Lujain Alrabghi ${ }^{1}$, Abdullah Alharbi ${ }^{2}$, \\ Ezdehar Alrowaithi ${ }^{2}$, Fatimah Almuallem ${ }^{3}$, Elaf Fakeih ${ }^{3}$, Bassmah Alrowaithi ${ }^{4}$, Hassan Allam ${ }^{5}$
}

${ }^{1}$ College of Medicine, King Abdulaziz University, Jeddah, Saudi Arabia

${ }^{2}$ College of Medicine, Ibn Sina National College, Jeddah, Saudi Arabia

${ }^{3}$ College of Medicine, Umm Al-Qura University, Mecca, Saudi Arabia

${ }^{4}$ Department of Obstetrics and Gynaecology, East Jeddah Hospital, Jeddah, Saudi Arabia

${ }^{5}$ Department of Obstetrics and Gynecology, Faculty of Medicine, King Abdulaziz University, Rabigh, Saudi Arabia

Received: 16 June 2018

Accepted: 20 June 2018

\section{*Correspondence:}

Dr. Maha Alhainiah,

E-mail: maha.hussain.94@gmail.com

Copyright: () the author(s), publisher and licensee Medip Academy. This is an open-access article distributed under the terms of the Creative Commons Attribution Non-Commercial License, which permits unrestricted non-commercial use, distribution, and reproduction in any medium, provided the original work is properly cited.

\section{ABSTRACT}

Uterine fibroid is one of the most common intrauterine masses among females at the reproductive age. Pregnancy and uterine fibroids are highly correlated. Pregnancy-related hormones influence the size of uterine fibroids, and fibroids have many impacts on pregnancy. Although most if the uterine fibroids are asymptomatic during pregnancy, serious complications may occur. The main complications include abortion, premature rupture of membranes, premature labor, abruptio placentae, peripartum hemorrhage, fetal malpresentation, fetal intrauterine growth retardation, small for gestational age infants, and fetal anomalies. The main risk factors for complications are related to the fibroid number, size, volume, location, and type. Large, multiple, retroplacental, submucosal, subserosal, pedunculated, or low-lying fibroids carries the highest risk for complications during pregnancy. This review will address the prevalence of uterine fibroids during pregnancy, its effects, and complications.

Keywords: Complications, Effect, Fibroid, Pregnancy, Safety, Uterine fibroid

\section{INTRODUCTION}

Uterine fibroid (also known as leiomyomas) is one of the most common intrauterine masses prevalent among women in reproductive age. It is estimated that it affects $40-60 \%$ of women at the age of 35 years and up to $80 \%$ of women at the ae of $50 .^{1}$

Uterine fibroids are benign tumors arising from the smooth muscles of the uterus. Although most of the uterine fibroids are asymptomatic, women begin to complain of the them when they increase in size or develop any complications, and the hormonal changes during pregnancy are one of the main conditions that lead increase the fibroid size. ${ }^{2}$ During pregnancy, the diagnosis of fibroids remains challenging. Less than one half of the large fibroids (42\%), and less than one eighth of the small fibroids $(12.5 \%)$ can be palpated by physical examination. ${ }^{3}$ Furthermore, ultrasound examination only reveals $1.4-2.7 \%$ of uterine fibroids. ${ }^{4}$

The effect and safety of uterine fibroids on pregnancy has been a topic of concern for decades. Researchers report conflicting opinions about this issue.

Some studies indicate that most of the fibroids are asymptomatic during pregnancy and are associated to safe uneventful pregnancy and labor, whilst others argue that uterine fibroids are sometimes associated with deleterious complications during pregnancy and/or labor 
and, therefore, should be managed preconceptionally. This article will review the prevalence, effects and complications, and safety of uterine fibroids on pregnancy.

\section{Prevalence of uterine fibroids among pregnant women}

The exact prevalence of uterine fibroids is not accurately determined. This is partly due to the asymptomatic nature of the vast majority of the fibroids, and partly due to the difficult diagnosis as previously mentioned. However, the prevalence of uterine fibroids during pregnancy reported in some studies ranges from 1.6 to $10.7 \%$, and the prevalence varies from one semester to another. ${ }^{1,5}$ The first trimester of pregnancy has the highest prevalence rate because some fibroids increase in size during this semester. ${ }^{6}$

On the other hand, in an attempt to explore the effect of uterine fibroids on infertility rates, the incidence of fibroids was found to range from 12 to $25 \%$ among women with infertility. However, a direct causal-effect relationship between fibroids and infertility could not be established. ${ }^{7,8}$

\section{Diagnosis of uterine fibroids among pregnancy}

During pregnancy, the vast majority of fibroids remain asymptomatic. Symptoms occur when the fibroid increases in size or develop complications. When uterine fibroids expand in size, they exert a mass effect on their blood vessels supply leading to ischemia, anoxia, and necrosis. Cell necrosis and damage are associated with increased prostaglandin release and subsequently localized pain. Another cause of necrosis is the large expansion of fibroid in size without a similar increase in blood flow, leading to a mismatch between the vascular demand of the tissue and the available blood supply. ${ }^{9}$ Furthermore, severe localized pain also arises from torsion of pedunculated sub-serosal uterine fibroids or from impaction of uterine fibroids compromising adjacent blood vessels. ${ }^{3}$ Therefore, pain is the most common presentation of large $(>5 \mathrm{~cm})$ or complicated uterine fibroids during pregnancy. ${ }^{7,9}$ Other less common symptoms include pelvic pressure, vaginal bleeding, urinary frequency, and constipation. ${ }^{7,10}$ The large-sized fibroids exert pressure effects on nearby organs as well. Anterior cervical fibroids compress the urinary bladder leading to frequent micturition. Sometimes, partial obstruction may result if compression occurred on bladder neck leading to urine retention.

Physical examination of uterine fibroids has a limited value. Only less than half of the large fibroids $(>5 \mathrm{~cm})$ can be revealed on physical examination. ${ }^{4}$ Smaller fibroids $(3-5 \mathrm{~cm})$ have only a $12.5 \%$ chance to be detected by physical examination. ${ }^{3}$ Ultrasonography can diagnose only $1.4-2.7 \%$ of uterine fibroids during pregnancy. Fibroids appear on ultrasonography as spherical mass lesions that are echogenically- distinguishable from surrounding uterine myometrium. ${ }^{11}$ The size, site, number, and some complications are assessed by ultrasound examination. Some ultrasonographic features of the tumor were reported to be associated with the clinical presentation. For instance, Leav-Toaf et al, in their ultrasonographic study on 113 pregnant women, found that among the 10 women who had cystic changes or heterogenous echogenic pattern of their fibroid, $7(70 \%)$ had severe localized abdominal pain. ${ }^{12}$ And in comparison, with the $11.7 \%$ (12 out of 103) who had abdominal pain without cystic changes or heterogenous echogenic patterns, the differences were statistically significant $(\mathrm{p}<0.05) .{ }^{12}$

\section{Effect of pregnancy on uterine fibroids}

The main effect of pregnancy on fibroid is related to the size of the uterine fibroids. It has been argued for many decades whether the hormonal changes occurring during pregnancy can influence the size of uterine fibroids or not. For a couple of decades, uterine fibroids were thought to enlarge during pregnancy, particularly during the first trimester of pregnancy. Many studies and review articles agreed with this theory. Benaglia et al, in their prospective cohort study on 25 women with fibroids and 25 controls, reported that the size of the fibroids increased dramatically (more than double the original size) during the first 7 weeks of gestations. ${ }^{13}$ Similarly, Rosati et al. reported a significant increase in size of uterine fibroids (of more than $8 \%$ ) during early pregnancy $(p<0.001) .{ }^{14}$ Similarly, Neiger et al. indicated that the pre-pregnant size of uterine fibroid was about $88 \%$ of the pregnant size among the studied women. ${ }^{15}$

The pregnancy-related size growth of uterine fibroids is largely attribute to the hormonal changes occurring during pregnancy. Estrogen is claimed to be the principle hormone promoting uterine growth. ${ }^{16}$ Progesterone is thought to be responsible for maintain the size of these tumors. ${ }^{17}$ However, sex hormone did not seem to be the only hormones involved. This is because the growth of uterine fibroid does not follow the same pattern of progressively increasing secretion of estrogen and progesterone i.e. a linear relationship between fibroid growth rate and sex hormone secretion had never been established. ${ }^{14,16}$ Although estrogen and progesterone increase steadily during pregnancy, fibroids shrink in size during second and third trimesters. ${ }^{12,14,16,18}$

Therefore, authors argue a role of other hormones in fibroid growth during pregnancy particularly human chorionic gonadotropin (hCG). ${ }^{19}$ The rapid increase in serum hCG occurring during the initial pregnancy may be responsible for the initial increase in size of the uterine fibroid noted during early pregnancy.

On the other hand, some studied reported that up to $78 \%$ of uterine fibroids do not show significant growth during pregnancy. ${ }^{14,20}$ The remaining $\sim 22 \%$ increased $12.5-25 \%$ of their initial size during the first 10 weeks of gestation. 


\section{Effects of uterine fibroids on pregnancy}

The impact of uterine fibroids on pregnancy has been a matter of concern for years. Although most of the uterine fibroids are asymptomatic and result in uneventful pregnancy and labor, complications are not uncommon. Pain is often the presenting symptom that warrants searching for uterine fibroids complications. This section will review the mechanism of effects, the complications, and safety of uterine fibroids on pregnancy

\section{Mechanism of uterine fibroid effects on pregnancy}

The mechanisms whereby uterine fibroids may lead to complications are not fully established. It is argued that the dramatic increase in size lead to compromising the blood supply and subsequent ischemic necrosis of the tumor. This results in pain, vaginal bleeding, and pelvic heaviness. The prostaglandin release resulting from tissue damage results in further increase in pain and uterine contractions. $^{7,9}$

Other mechanisms involved include mechanical effects of uterine fibroids on fetal sac. The size, site, number, type (subserosal, intramural, or submucosal), and shape (sessile or pedunculated) are responsible for various mechanisms of adverse effects. ${ }^{4}$ For instance, subserosal fibroids are at risk for torsion and red degeneration, lower-located fibroids (i.e. near cervix) might result in obstructed labor, submucosal fibroids are associated with higher miscarriage rates, and large-sized fibroids $(>5 \mathrm{~cm})$ are associated with uterine cavity distortion, abnormal fetal position, and presentation. ${ }^{21-23}$.

\section{Complications}

Though rare, uterine fibroids are associated with significantly higher risk of complications during pregnancy and labor when compared to healthy women. Uterine fibroids, particularly those of submucosal origin, are associated with high risk of abnormal fetal position and presentation. It is estimated that uterine fibroids are associated with $13 \%$ risk for breech presentation, $19 \%$ risk for pre-term labor and premature rupture of membranes, $49 \%$ risk for cesarean delivery, and $8 \%$ risk for abortion, antenatal hemorrhage, postpartum hemorrhage, abruptio placentae, and labor dystocia. ${ }^{23}$

Abortion is often associated with submucosal fibroids. It is estimated to occur in $14 \%$ of pregnant females with fibroids in comparison to $7.6 \%$ in healthy pregnant women. The main determinant for abortion is the location of the fibroid. Abortion was reported to be 8 times more common among pregnant ladies with retroplacental fibroids than those with non-retroplacental fibroids. ${ }^{24}$ Abruptio placentae occurs due to impaired blood flow to the placenta and adjacent uterine myometrium. Therefore, it often with submucous, large $(>7.8 \mathrm{~cm}$ or $>200 \mathrm{ml}$ volume), or retroplacental fibroids. ${ }^{25}$ Preterm labor and premature rupture of membranes (PROM) are other significant complications of uterine fibroid. They are associated with retroplacental large fibroids (3.6 $\mathrm{cm}) .{ }^{12,14,24}$. Although significant, preterm labor was estimated to be only 2 or 3 weeks prior to the expected date of delivery which does not have a significant impact on the fetus. ${ }^{26}$

Other complications include fibroid torsion and red degeneration, fetal anomalies, fetal growth restriction, fetal demise, and preeclampsia. Fibroid torsion often occurs during the first trimester when there is an adequate intrauterine space for peduncular twist. It results from pedunculated subserosal types of uterine fibroid. The main risk factor for uterine fibroid torsion is rapid body movements. Red degeneration results from this torsion and is estimated to have a prevalence of $5 \%$ during pregnancy. ${ }^{27}$ It presents clinically with severe abdominal pain, nausea, vomiting, fever, local tenderness and rebound tenderness. ${ }^{8}$

Fetal lie and malpresentation are common consequences of uterine distortion. Breech presentation and oblique lie are the most common abnormalities encountered; and they are associated with large $(>5 \mathrm{~cm})$, multiple, and lower-lying fibroids. ${ }^{28}$ Other reported fetal complications reported are small for gestational age infants, intrauterine growth retardation, and rarely fetal anomalies. The fetal anomalies noted with uterine fibroids were congenital torticollis, head deformities, and limb reduction defects. $^{26,27}$

\section{CONCLUSION}

Pregnancy and uterine fibroids are highly correlated. Pregnancy-related hormones influence the size of uterine fibroids, and fibroids have many impacts on pregnancy. Although most if the uterine fibroids are asymptomatic during pregnancy, serious complications may occur. The main complications include abortion, premature rupture of membranes, premature labor, abruptio placentae, peripartum hemorrhage, fetal malpresentation, fetal intrauterine growth retardation, small for gestational age infants, and fetal anomalies. The main risk factors for complications are related to the fibroid number, size, volume, location, and type. Large, multiple, retroplacental, submucosal, subserosal, pedunculated, or low-lying fibroids carries the highest risk for complications during pregnancy.

Funding: No funding sources

Conflict of interest: None declared

Ethical approval: Not required

\section{REFERENCES}

1. Baird DD, Dunson DB, Hill MC, Cousins D, Schectman JM. High cumulative incidence of uterine leiomyoma in black and white women: Ultrasound evidence. Am J Obstet Gynecol. 2003;188(1):100-7. 
2. Johnson NL, Norwitz E, Segars JH. Management of Fibroids in Pregnancy. In: Fibroids. 2013:36-53.

3. Parker WH. Etiology, symptomatology, and diagnosis of uterine myomas. Fertil Steril. 2007;87(4):725-36

4. Nair S. Contemporary Management of Fibroids. Ann Acad Med Singapore. 2003;32(5):615-23.

5. Shavell VI, Thakur M, Sawant A, Kruger ML, Jones TB, Singh M, Puscheck EE, Diamond MP. Adverse obstetric outcomes associated with sonographically identified large uterine fibroids. Fertil Steril. 2012;97(1):107-10.

6. Cooper NP, Okolo S. Fibroids in pregnancy-common but poorly understood. Obstet Gynecol Surv. 2005;60(2):132-8.

7. Evans P, Brunsell S. Uterine fibroid tumors: Diagnosis and treatment. Am Fam Physician. 2007;75(10):1503-8.

8. Calaf J, Arque M, Porta O, D'Angelo E. (The fibroid as clinical problem). Med Clin (Barc). 2013;141 Suppl:1-6.

9. Vitale SG, Tropea A, Rossetti D, Carnelli M, Cianci A. Management of uterine leiomyomas in pregnancy: Review of literature. Updates Surg. 2013;65(3):17982.

10. Donnez J, Dolmans MM. Uterine fibroid management: From the present to the future. Hum Reprod Update. 2016;22(6):665-86.

11. Myers SL, Baird DD, Olshan AF, Herring AH, Schroeder JC, Nylander-French LA, Hartmann KE. Self-report versus ultrasound measurement of uterine fibroid status. J Women's Health. 2012;21(3):285-93.

12. Lev-Toaff S, Coleman BG, Arger PH, Mintz MC, Arenson RL, Toaff ME. Leiomyomas in pregnancy: sonographic study. Radiology. 1987;164(2):375-80.

13. Benaglia L, Cardellicchio L, Filippi F, Paffoni A, Vercellini P, Somigliana E, Fedele L, et al. The rapid growth of fibroids during early pregnancy. PloS one. 2014;9(1):e85933.

14. Rosati P, Exacoustos C, Mancuso S. Longitudinal evaluation of uterine myoma growth during pregnancy: A sonographic study. J Ultrasound Med. 1992;11(10):511-5.

15. Neiger R, Sonek JD, Croom CS, Ventolini G. Pregnancy-related changes in the size of uterine leiomyomas. J Reprod Med. 2006;51:671-4.

16. De Vivo A, Mancuso A, Giacobbe A, et al. Uterine myomas during pregnancy: A longitudinal sonographic study. Ultrasound Obstet Gynecol. 2011;37(3):361-5.
17. Shikawa H, Ishi K, Ann Serna V, Kakazu R, Bulun SE, Kurita T. Progesterone is essential for maintenance and growth of uterine leiomyoma. Endocrinology. 2010;151(6):2433-42.

18. Hammoud AO, Asaad R, Berman J, Treadwell MC, Blackwell S, Diamond MP. Volume change of uterine myomas during pregnancy: Do myomas really grow? J Minim Invasive Gynecol. 2006;13(5):386-90.

19. Horiuchi A, Nikaido T, Yoshizawa T, et al. HCG promotes proliferation of uterine leiomyomal cells more strongly than that of myometrial smooth muscle cells in vitro. Mol Hum Reprod. 2000;6(6):523-8.

20. Aharoni A, Reiter A, Golan D, Paltiely Y, Sharf M. Patterns of growth of uterine leiomyomas during pregnancy. A prospective longitudinal study. Br J Obstet Gynaecol. 1988;95(5):510-3.

21. Akinyemi BO, Adewoye BR, Fakoya TA. Uterine fibroid: a review. Niger J Med. 2004;13(4):318-29.

22. Klatsky PC, Tran ND, Caughey AB, Fujimoto VY. Fibroids and reproductive outcomes: a systematic literature review from conception to delivery. Am J Obstet Gynecol. 2008;198(4):357-66.

23. Zaima A, Ash A. Fibroid in pregnancy: Characteristics, complications, and management. Postgrad Med J. 2011;87(1034):819-28.

24. Winer-Muram HT, Muram D, Gillieson MS, Ivey BJ, Muggah HF. Uterine myomas in pregnancy. Can Med Assoc J. 1983;128(8):949-50.

25. Xacoustos C, Rosati P. Ultrasound diagnosis of uterine myomas and complications in pregnancy. Obs Gynecol. 1993;82:97-101.

26. Navid S, Arshad S, Qurat-ul-Ain, Meo RA. Impact of leiomyoma in pregnancy. J Ayub Med Coll Abbottabad. 2012;24(1):90-2.

27. Sparić R. Uterine myomas in pregnancy, childbirth and puerperium. Srp Arh Celok Lek. 2014;142(12):118-24.

28. Hasan F, Arumugam K, Sivanesaratnam V. Uterine leiomyomata in pregnancy. Int $\mathbf{J}$ Gynecol Obstet. 1991;34(1):45-8.

Cite this article as: Alhainiah M, Aljifry E, Alghamdi A, Alrabghi L, Alrabghi A, Alrowaithi E et al. Safety of pregnancy in uterine fibroids. Int J Reprod Contracept Obstet Gynecol 2018;7:2985-8. 